\title{
Inadvertent Error: Evaluation of Bevacizumab for the Treatment of Epidural Fibrosis by Immunohistochemical Staining for CD105 and Osteopontin
}

\author{
Ilhan YILMAZ ${ }^{1}$, Uzay ERDOGAN², Osman TANRIVERDI ${ }^{2}$, Omur GUNALDI ${ }^{2}$, H. Utku ADILAY², \\ Muhammed OMEROGLU ${ }^{4}$, Canan TANIK ${ }^{5}$, Bekir TUGCU² \\ ${ }^{1}$ University of Health Sciences, Sisli Hamidiye Etfal Training and Research Hospital, Department of Neurosurgery, Istanbul, Turkey \\ ${ }^{2}$ University of Health Sciences, Bakirkoy Prof. Dr. Mazhar Osman Training and Research Hospital for Neurology, Neurosurgery and Psychiatry, \\ Istanbul, Turkey \\ ${ }^{3}$ Balikesir University, School of Medicine, Department of Neurosurgery, Balikesir, Turkey \\ ${ }^{4}$ Toyotasa State Hospital, Department of Neurosurgery, Sakarya, Turkey \\ ${ }^{5}$ University of Health Sciences, Sisli Hamidiye Etfal Training and Research Hospital, Department of Pathology, Istanbul, Turkey
}

Corresponding author: Osman TANRIVERDI osmantanriverdi74@gmail.com

A $S$ the authors of the paper titled "Evaluation of Bevacizumab for the Treatment of Epidural Fibrosis by Immunohistochemical Staining for CD105 and Osteopontin" published in 2018, Volume 28(6), pp. 954-962, we have noticed after a retrospective review of this paper that Figure 3A is incorrect, namely, the picture of Figure 3B is correct but not the caption (1). The information regarding Figure 3A-B was explained as it should be within the text, but, mistakenly, the wrong picture and caption were provided in Figure 3A, and the wrong caption was given for Figure 3B. The (incorrect) version of Figure $\mathbf{3 A - B}$ published in the paper was as follows:
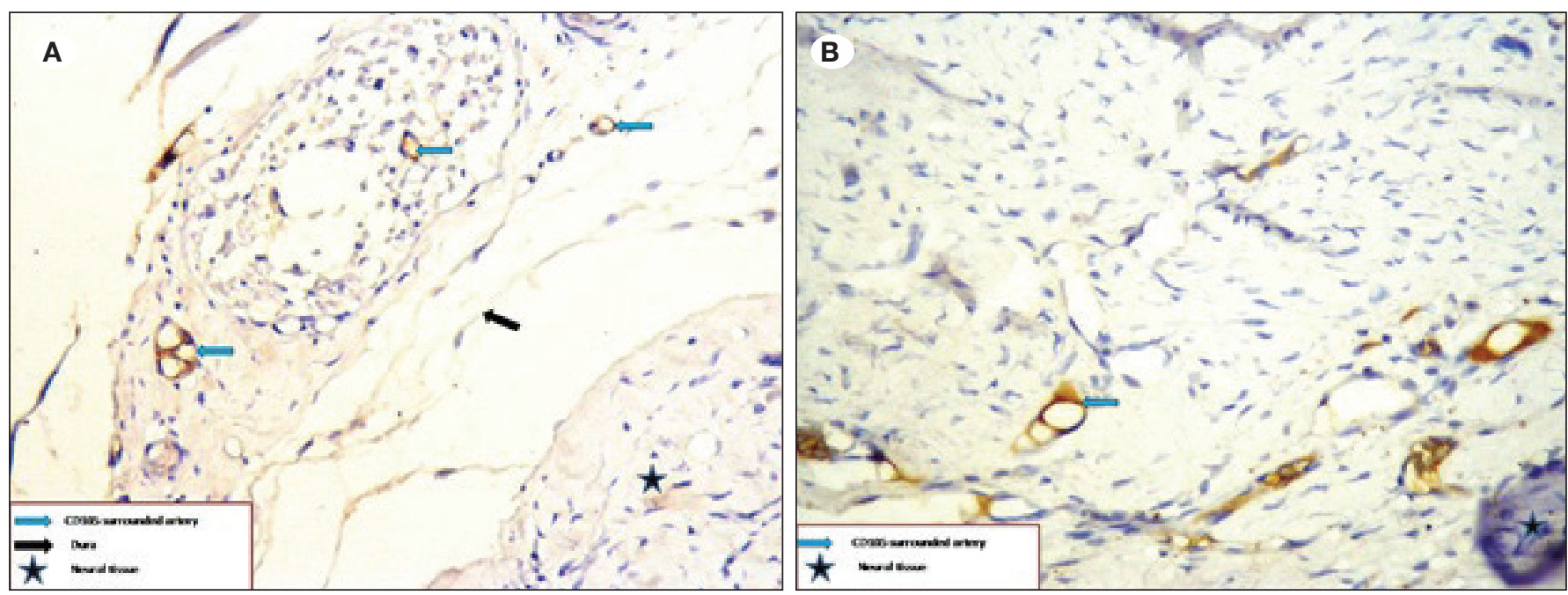

Figure 3: Immunohistochemical staining for CD105. A) Grade III fibrosis in a control animal in group I. B) Grade I fibrosis in a bevacizumabtreated animal in group II (star, neural tissue; black arrow, dura; blue arrow, fibrosis).

Ilhan YILMAZ

(1) : $0000-0001-5548-2228$

Uzay ERDOGAN

(D) : 0000-0001-8268-6306

Osman TANRIVERDI (1) : 0000-0002-4846-4213
Omur GUNALDI

H. Utku ADILAY

(D) : $0000-0003-4867-244 \mathrm{X}$

Muhammed OMEROGLU (D) : 0000-0001-7130-1802
Canan TANIK (1) : 0000-0003-2435-7533

Bekir TUGCU (D) : 0000-0003-0385-0054 
The (correct) pictures and captions of Figure 3A-B should be as follows:
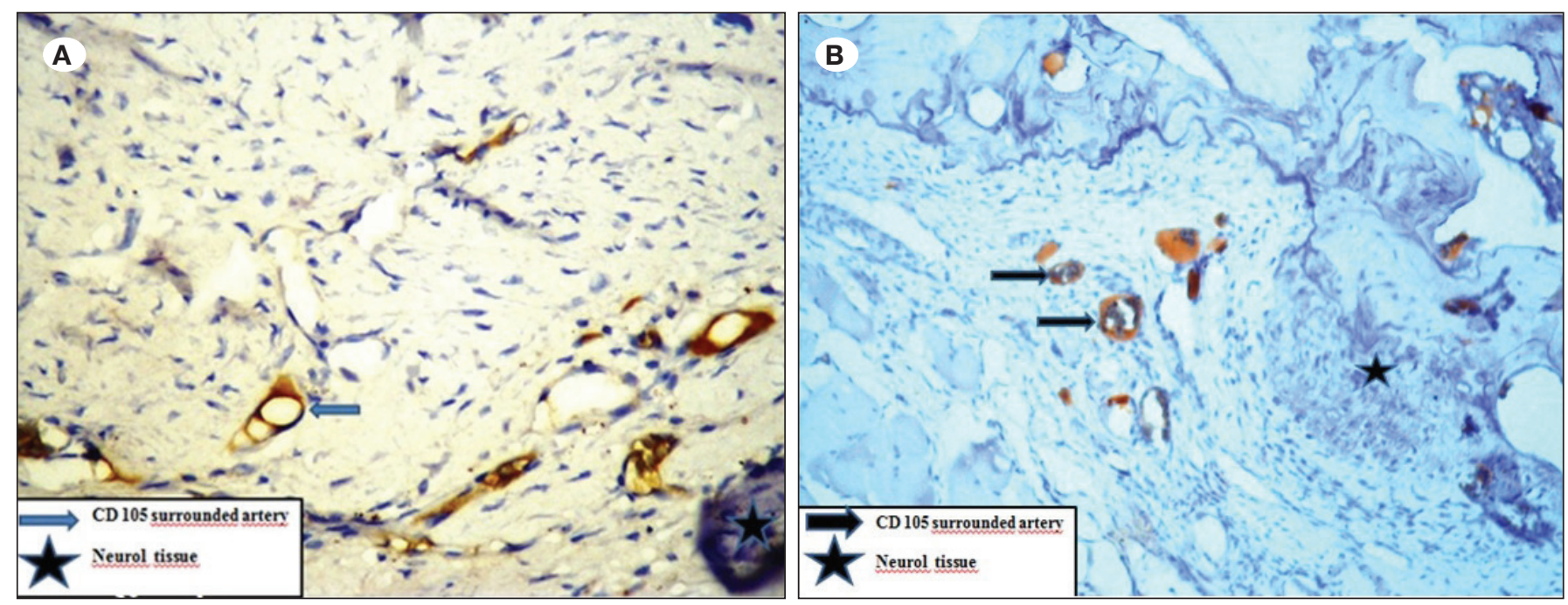

Figure 3: Immunohistochemical staining for CD105. A) Grade III fibrosis in a control animal in group I (star, neural tissue; blue arrow, fibrosis). B) Grade I fibrosis in a bevacizumab-treated animal in group II (star, neural tissue; black arrow, fibrosis).

The error relating to Figure $3 \mathbf{A}-\mathbf{B}$ in the published version did not affect any statistical data or the study outcome.

As the authors of the article, we hereby amend this mistake and correct the figures and present them as they should be in order not to misinform our colleagues who will read our study.

\section{REFERENCES}

1. Yilmaz I, Erdogan U, Tanriverdi O, Gunaldi O, Adilay U, Omeroglu M, Tanik C, Tugcu B: Evaluation of bevacizumab for the treatment of epidural fibrosis by immunohistochemical staining for CD105 and osteopontin. Turk Neurosurg 28(6):954962, 2018 